\title{
Effects of restrictive red blood cell transfusion on the prognoses of adult patients undergoing cardiac surgery: a meta-analysis of randomized controlled trials
}

\author{
Qi-Hong Chen ${ }^{1}$, Hua-Ling Wang ${ }^{2 *}$, Lei Liu', Jun Shao', Jiangqian Yu' and Rui-Qiang Zheng ${ }^{1}$
}

\begin{abstract}
Purpose: Restrictive red blood cell transfusion strategies remain controversial in patients undergoing cardiac surgery. We performed a meta-analysis to assess the prognostic benefits of restrictive red blood cell transfusion strategies in patients undergoing cardiac surgery.
\end{abstract}

Methods: We identified randomized clinical trials through the 9th of December 2017 that investigated a restrictive red blood cell transfusion strategy versus a liberal transfusion strategy in patients undergoing cardiac surgery. Individual patient data from each study were collected. Meta-analyses were performed for the primary and secondary outcomes. The risk of bias was assessed using the Cochrane Risk of Bias Tool. A trial sequential analysis (TSA)-adjusted random-effects model was used to pool the results from the included studies for the primary outcomes.

Results: Seven trials involving a total of 8886 patients were included. The TSA evaluations suggested that this meta-analysis could draw firm negative results, and the data were sufficient. There was no evidence that the risk of 30-day mortality differed between the patients assigned to a restrictive blood cell transfusion strategy and a liberal transfusion strategy (odds ratio (OR) $0.98 ; 95 \%$ confidence interval $(\mathrm{Cl}) 0.77$ to $1.24 ; p=0.87$ ). Furthermore, the study suggested that the restrictive transfusion strategy was not associated with significant increases in pulmonary morbidity (OR 1.09; 95\% Cl 0.88 to 1.34; $p=0.44$ ), postoperative infection (OR $1.11 ; 95 \% \mathrm{Cl} 0.95$ to $1.3 ; p=0.58)$, acute kidney injury (OR 1.03; $95 \% \mathrm{Cl} 0.92$ to $1.14 ; p=0.71)$, acute myocardial infarction (OR $1.01 ; 95 \% \mathrm{Cl} 0.80$ to 1.27; $p=0.78$ ), or cerebrovascular accidents (OR $0.97 ; 95 \% \mathrm{Cl} 0.72$ to $1.30 ; p=0.66$ ).

Conclusions: Our meta-analysis demonstrates that the restrictive red blood cell transfusion strategy was not inferior to the liberal strategy with respect to 30-day mortality, pulmonary morbidity, postoperative infection, cerebrovascular accidents, acute kidney injury, or acute myocardial infarction, and fewer red blood cells were transfused.

Keywords: Restrictive transfusion strategy, Liberal transfusion strategy, Cardiac surgery, Prognosis, Meta-analyses

\footnotetext{
* Correspondence: 18051063567@163.com

${ }^{2}$ Department of Cardiology, Northern Jiangsu People's Hospital; Clinical Medical College, Yangzhou University, 98 Nantong West Road, Yangzhou 225001, People's Republic of China

Full list of author information is available at the end of the article
}

(c) The Author(s). 2018 Open Access This article is distributed under the terms of the Creative Commons Attribution 4.0 International License (http://creativecommons.org/licenses/by/4.0/), which permits unrestricted use, distribution, and reproduction in any medium, provided you give appropriate credit to the original author(s) and the source, provide a link to the Creative Commons license, and indicate if changes were made. The Creative Commons Public Domain Dedication waiver (http://creativecommons.org/publicdomain/zero/1.0/) applies to the data made available in this article, unless otherwise stated. 


\section{Background}

Anemia is common after cardiac surgery and is associated with significant increases in morbidity and mortality [1-3]. Red blood cell (RBC) transfusions can be lifesaving in patients with severe anemia and the purpose of perioperative $\mathrm{RBC}$ transfusion is to improve oxygen delivery in patients with anemia [4]. More than $50 \%$ of patients receive a postoperative transfusion, which uses a substantial proportion of blood supplies [5].

However, RBC transfusion has been associated with high rates of mortality and morbidity in critically ill patients [6]. It is associated with infection, acute lung injury, acute kidney injury, and death [7]. The infectious and non-infectious risks associated with transfusion support restrictive transfusion practices in several clinical settings [8]. Whether the restrictive approach to preoperative $\mathrm{RBC}$ transfusion in cardiac surgery safely achieves outcomes similar to those achieved by means of more liberal approaches remains unclear.

Recent studies have demonstrated that a restrictive strategy for RBC transfusion is not inferior to a liberal strategy with respect to death and other outcomes in patients undergoing cardiac surgery $[9,10]$. The aim of this meta-analysis is to assess the effects of restrictive compared to liberal RBC transfusion on the prognoses of adult patients undergoing cardiac surgery.

\section{Methods}

\section{Eligibility criteria}

We included trials with the following features:

1. Types of studies: Randomized controlled clinical trials

2. Population: Patients undergoing cardiac surgery

3. Intervention: Patients receiving restrictive $\mathrm{RBC}$ transfusion

4. The following outcomes were included: a) primary outcome, 30-day mortality; b) secondary outcomes, pulmonary morbidity (including acute respiratory distress syndrome, acute lung injury, delayed extubation), postoperative infection (including deep sternal wound infection, leg wound infection, sepsis, etc.), cerebrovascular accident, acute kidney injury (including all stages, acute kidney injury requiring renal replacement treatment), and myocardial infarction.

\section{Search strategy and study selection}

We searched the Medline, Elsevier, Embase, Cochrane (Central), Web of Science, and ClinicalTrials.gov databases from inception to December 9, 2017 for studies investigating the perioperative use of restrictive $\mathrm{RBC}$ transfusion in patients undergoing cardiac surgery. Two reviewers independently reviewed all abstracts and titles and excluded trials that were obviously irrelevant. The full texts of the articles were then reviewed independently in accordance with the inclusion and exclusion criteria. Any discrepancies were resolved by reaching a consensus regarding the inclusion or exclusion of a trial by discussion with a third reviewer.

\section{Data extraction and management}

Two reviewers independently extracted the data using a standardized data extraction protocol. Any disagreements between the two reviewers were resolved by discussion. Information, including trial characteristics, included authors, year of publication, country of origin, study design, sample size, the inclusion and exclusion criteria, the methods of statistical adjustment, transfusion strategies, and study results, was extracted from the included studies.

\section{Trial sequential analysis}

We conducted a trial sequential analysis (TSA) to prevent the risk of increases in random error by repeated updates according to the method we described previously [11]. A TSA-adjusted random-effects model was used to pool the results from the included studies for the primary outcomes. A two-sided TSA was performed to maintain a risk of $5 \%$ for type I error and a power of $80 \%$. Additionally, an estimated function was used to calculate the required information size.

\section{Statistical analysis}

Review Manager (version 5.3) was used for the meta-analysis. For each of the included studies, we calculated the odds ratio (OR) with 95\% confidence intervals (CIs) for dichotomous outcomes. The heterogeneity among studies was calculated with the Mantel-Haenszel chi-square test and the $\mathrm{I}^{2}$ test. The statistical heterogeneity of the data was quantified. Obvious heterogeneity was defined as $p<0.05$ using the Mantel-Haenszel chi-square test or an $\mathrm{I}^{2}>50 \%$. Furthermore, the funnel plot technique was used to assess the publication bias.

\section{Results \\ Study location and selection}

Our search strategy identified a total of 6765 titles and abstracts. After screening the abstracts and title, 4535 publications were left after duplicates were removed. Among them, 4431 publications were non-relevant, which were therefore excluded. The remaining 104 publications were retrieved for an eligibility assessment; 97 publications were deemed ineligible and were therefore excluded. Seven studies with a total of 8886 patients were included in the final analysis [9, 10, 12-16] (Fig. 1). 


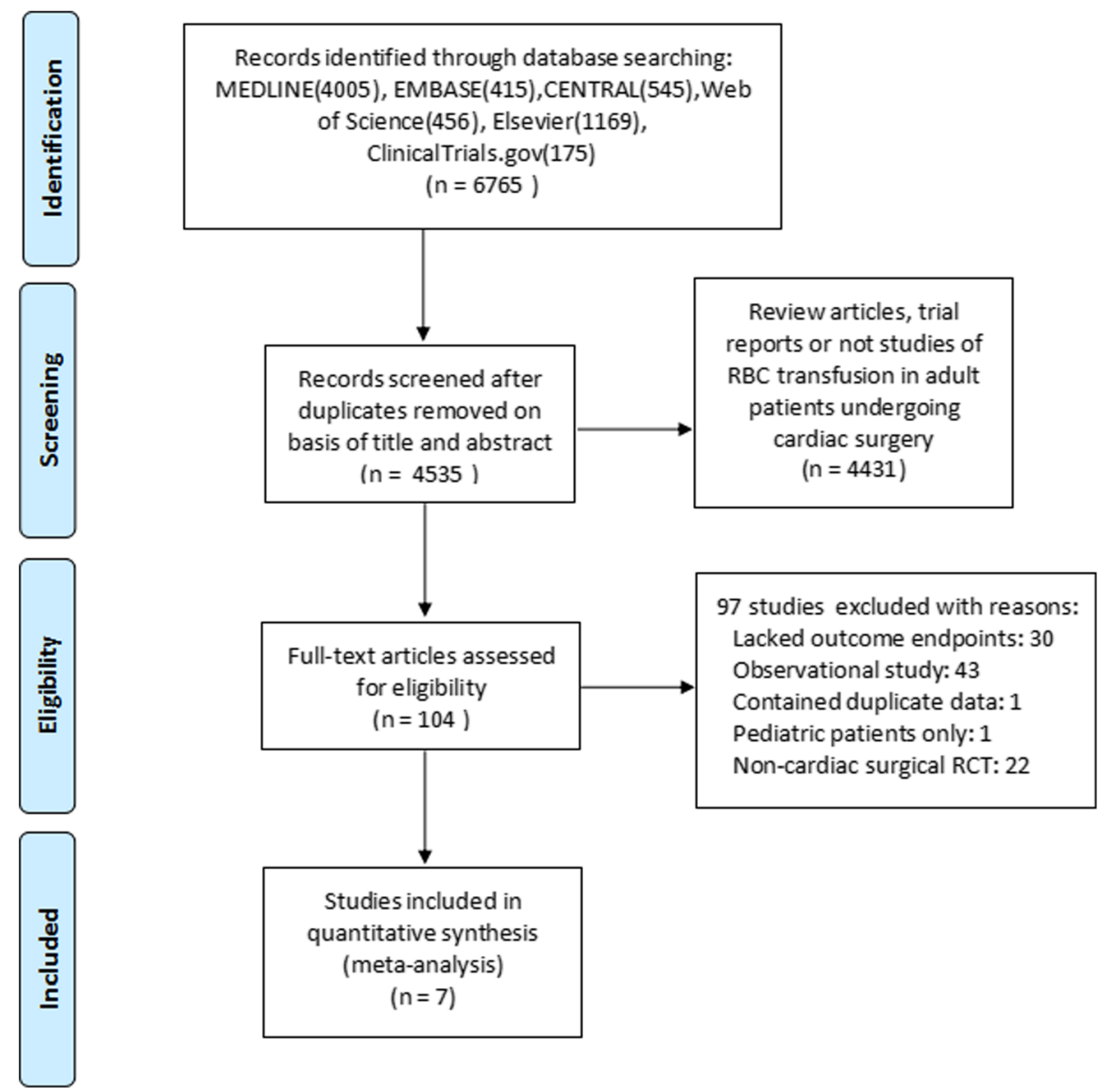

Fig. 1 Flow diagram of the identified trials. $R C T$ randomized controlled trial

\section{Characteristics of the trials}

We included seven trials that compared restrictive $\mathrm{RBC}$ transfusion with controls in patients undergoing cardiac surgery. The characteristics of the included trials are presented in Table 1. Four trials included only low-risk surgical patients who were undergoing elective cardiac surgery and excluded patients who were at the highest risk of requiring $\mathrm{RBC}$ transfusion $[10,12,13,15]$. The other three trials included patients who were at the highest risk of requiring $\mathrm{RBC}$ transfusion $[9,14,16]$. Patients allocated to the restrictive $R B C$ transfusion group were infused with fewer RBCs compared to patients in the liberal-threshold group. The median number of cell salvage and allogeneic RBC units transfused per patient ranged from one to three in the four studies [9, 1214]. $\mathrm{RBC}$ transfusion rates reported in three trials ranged from 44 to $75 \%[10,13,15]$. The other trial did not report the units of RBC transfusion or transfusion rate [7]. The results of random sequence generation are shown in Fig. 2.

\section{Trial sequential analysis}

A TSA sensitivity analysis including all trials revealed that the diversity-adjusted information size was 8886 patients. The cumulative z-curve did not cross the conventional boundary for benefit or the trial sequential monitoring boundary for benefit but did cross the estimated information size boundary (Fig. 3). The TSA evaluations suggested that this meta-analysis could draw firm negative results, and the data were sufficient.

\section{Mortality}

The effect of restrictive RBC transfusion on 30-day mortality rates was estimated from seven trials that included a total of 8886 patients. A total of 139 deaths occurred among 4440 patients who were allocated to the restrictive $\mathrm{RBC}$ transfusion group compared with 142 deaths among the 4446 patients allocated to the control group. No evidence of publication bias was detected after a funnel plot analysis (Fig. 4), and the heterogeneity was determined to be non-significant $\left(p=0.36, \mathrm{I}^{2}=9\right)$. There was no evidence that the risk of 30-day mortality differed between the patients assigned to the restrictive $\mathrm{RBC}$ transfusion and control groups (OR 0.98; 95\% CI 0.77 to $1.24 ; p=0.87$; Fig. 5 ). 


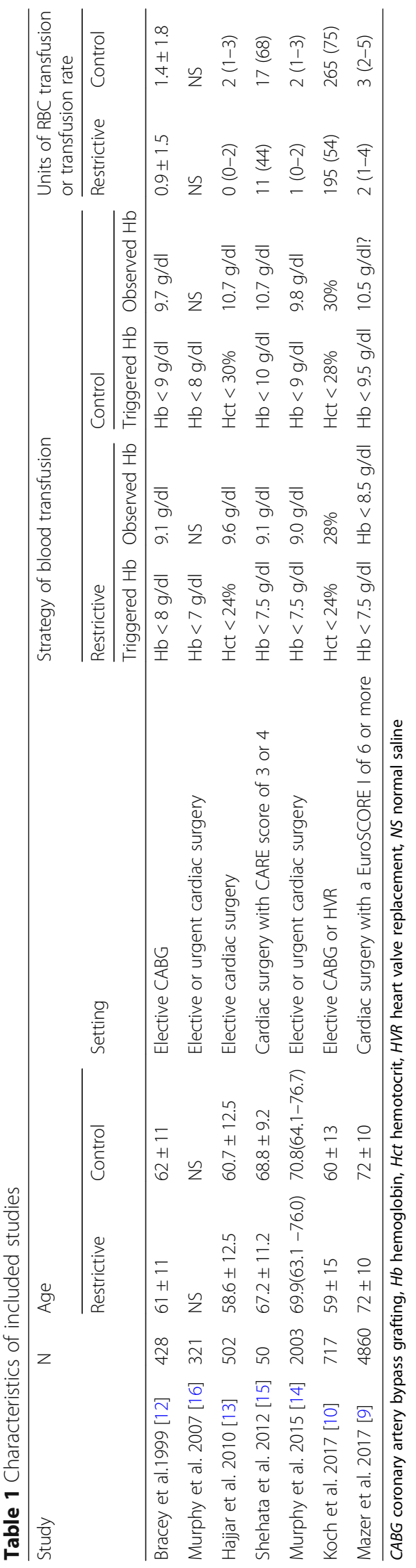




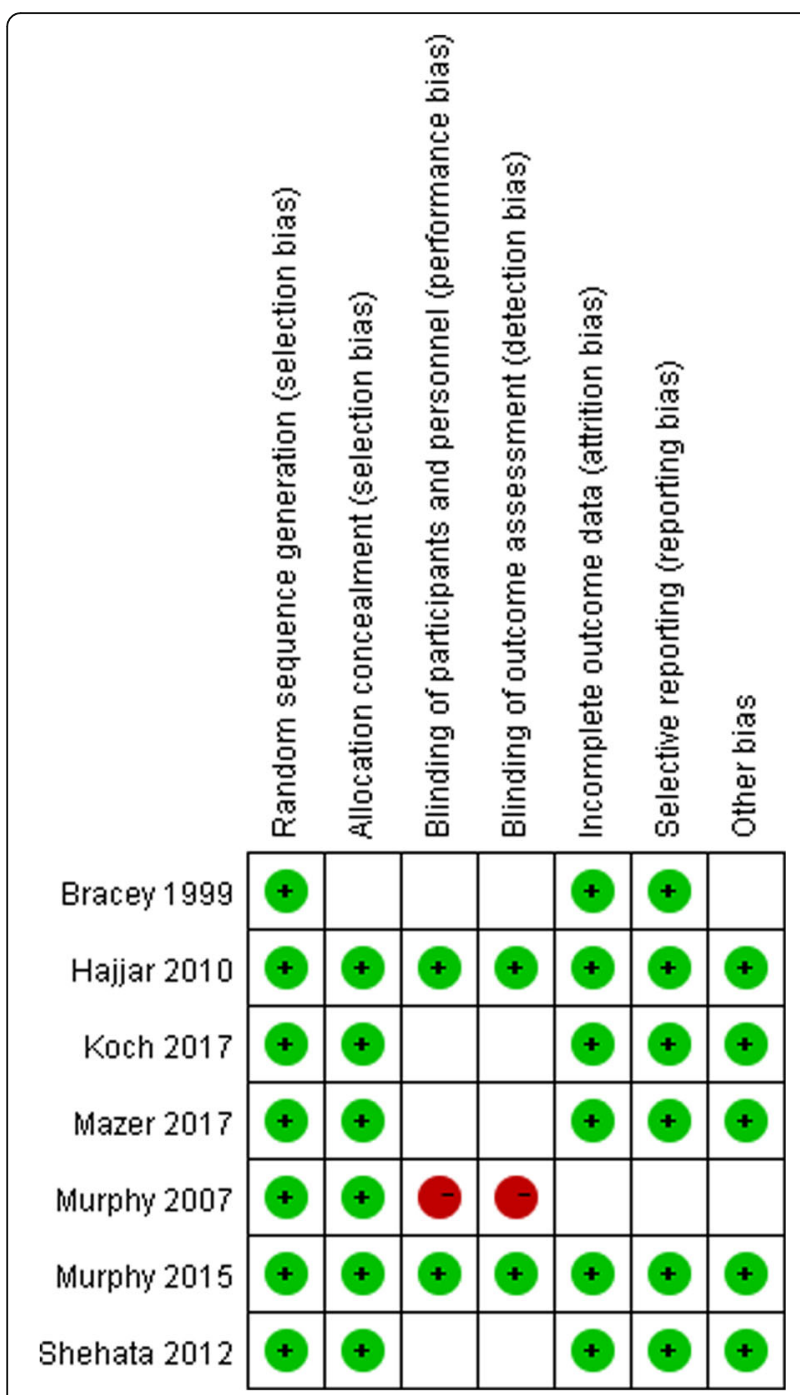

Fig. 2 Risk of bias summary. Review of the authors' judgements about each risk of bias item for each included study. Red indicates high risk, green indicates low risk, blank indicates unclear

\section{Secondary outcomes}

Five studies (3658) reported pulmonary morbidity as an outcome. The results revealed that there was no significant reduction in the risk of pulmonary morbidity with restrictive $\mathrm{RBC}$ transfusion ( $p=0.42$, Table 2$)$. Furthermore, the study suggested that the restrictive transfusion strategy was not associated with significant increases in pulmonary morbidity, postoperative infection, acute kidney injury, acute myocardial infarction, or cerebrovascular accidents (Table 2, Additional files 1, 2, 3, 4, and 5).

\section{Discussion}

Restrictive RBC transfusion strategies remain controversial in patients undergoing cardiac surgery [3, 7]. Thus, the effect of restrictive versus liberal transfusion strategies on clinical outcomes in patients undergoing cardiac surgery remains to be defined. Our meta-analysis demonstrated that the OR for 30-day mortality did not favor a restrictive transfusion strategy or a liberal transfusion strategy in randomized controlled trials of adult patients undergoing cardiac surgery. Furthermore, a restrictive RBC transfusion strategy was not inferior to a liberal strategy with respect to pulmonary morbidity, postoperative infection, cerebrovascular accident, acute kidney injury, or acute myocardial infarction, and fewer RBCs were transfused.

Some studies have suggested that the transfusion of RBCs is associated with many harmful effects, such as infection, acute lung injury, acute kidney injury, prolonged hospital stays, and increased mortality and hospital costs [7, 17]. A restrictive threshold for transfusion is likely to be favored because it requires the use of fewer units of RBCs $[18,19]$. Considering the known risks of $\mathrm{RBC}$ transfusions and the observational studies linking transfusion with increased adverse complications [20], clinicians have been adopting restrictive RBC transfusion strategies in cardiac surgery [21]. However, restrictive $\mathrm{RBC}$ transfusion strategies remain controversial in patients undergoing cardiac surgery [22]. Patients undergoing cardiac surgery have a lower cardiovascular reserve and restrictive $\mathrm{RBC}$ transfusion may increase the risk of anemia-induced tissue hypoxia [23]. Our meta-analysis provides evidence that restrictive transfusion is not associated with the risk of adverse outcomes such as infection, acute kidney injury, and pulmonary morbidity. However, the definitions of those secondary outcomes differed between studies. For instance, the KDIGO criteria were adopted to diagnose acute kidney injury in TRICS 3 trial [9], but Hajjar et al. applied the RIFLE classification [13], and some others employed dialysis-dependent or $50 \%$ or greater increase in serum creatinine $[15,16]$. Nonetheless, this meta-analysis suggests that restrictive transfusion strategies are as safe as liberal strategies in patients undergoing cardiac surgery.

Observational studies of adult patients undergoing cardiac surgery have shown strong associations between $\mathrm{RBC}$ transfusion and high mortality $[24,25]$. In the Transfusion Indication Threshold Reduction (TITRe2) clinical trial, 90-day mortality was higher with restrictive postoperative RBC transfusion than with a liberal threshold [14]. A meta-analysis of there randomized controlled trails reported that the odds for mortality favored a liberal RBC transfusion strategy rather than a restrictive $\mathrm{RBC}$ transfusion strategy, but the difference between strategies was not statistically significant [20]. However, the recently published TITRe3 trial did not provide evidence supporting this. The study showed that in patients undergoing cardiac surgery who were at moderate to high risk for death, a restrictive RBC transfusion strategy was noninferior to a liberal strategy with 


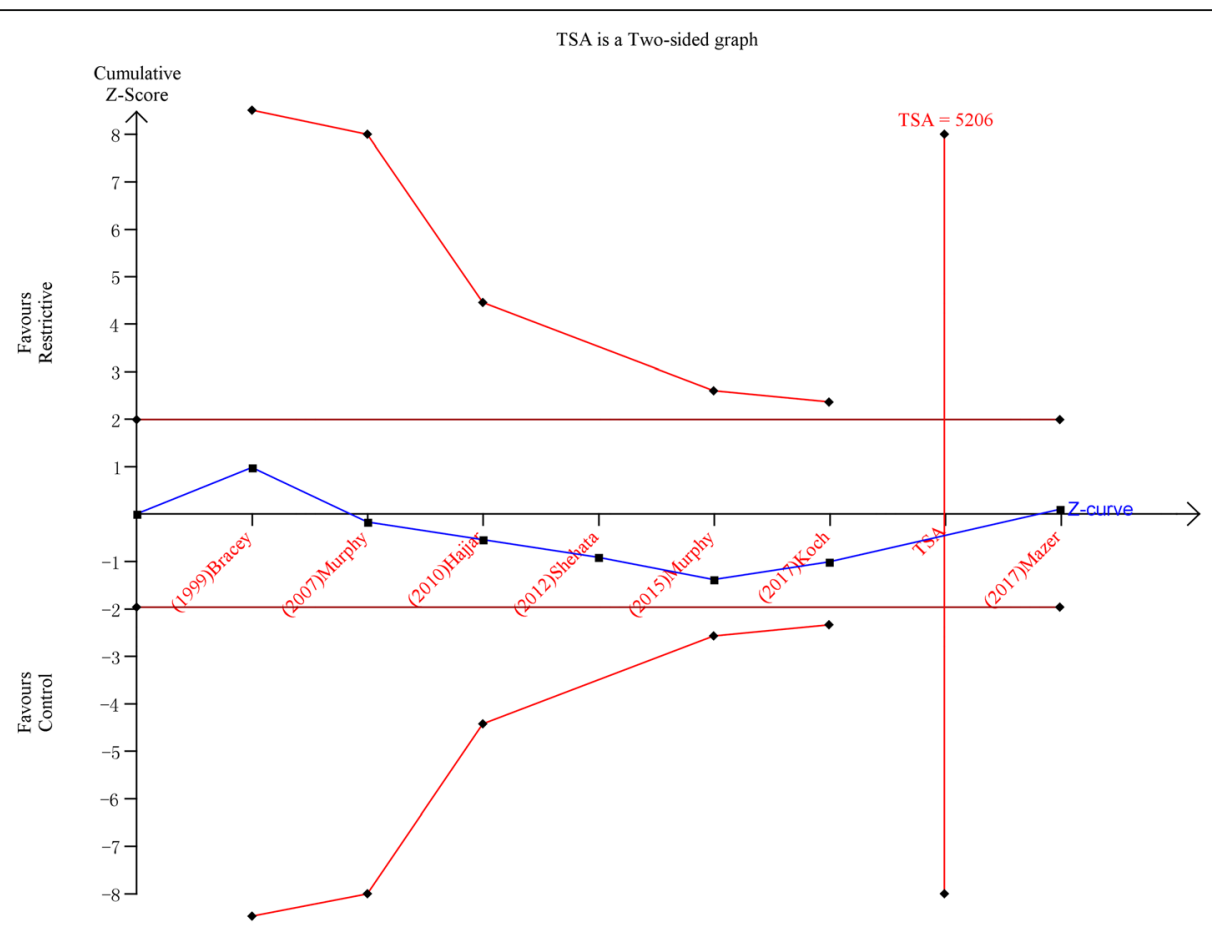

Fig. 3 Trial sequential analysis for mortality in the randomized controlled trials with a two-sided boundary and an incidence of $2.78 \%$ in the control arm and an incidence of $1.42 \%$ in the treatment arm

respect to the composite outcome of death from any cause [9]. Similar to the TRICS 3 trial, our meta-analysis demonstrated that a restrictive RBC transfusion strategy is not inferior to a liberal strategy with respect to 30-day mortality. To avoid the risk of random error increase due to repeated updates, a sensitivity analysis of the TSA was performed. The TSA evaluations suggested that this meta-analysis could draw firm negative results, and the data were sufficient. Thus, the restrictive RBC transfusion strategy was not inferior to the liberal strategy with respect to 30-day mortality.

There are some procedures and techniques to reduce $\mathrm{RBC}$ transfusion in patients undergoing cardiac surgery [26]. In 2010, the World Health Organization encouraged all member countries to implement patient blood management (PBM) programs employing multiple combined strategies to increase and preserve autologous erythrocyte volume to restrict RBC transfusions [27].

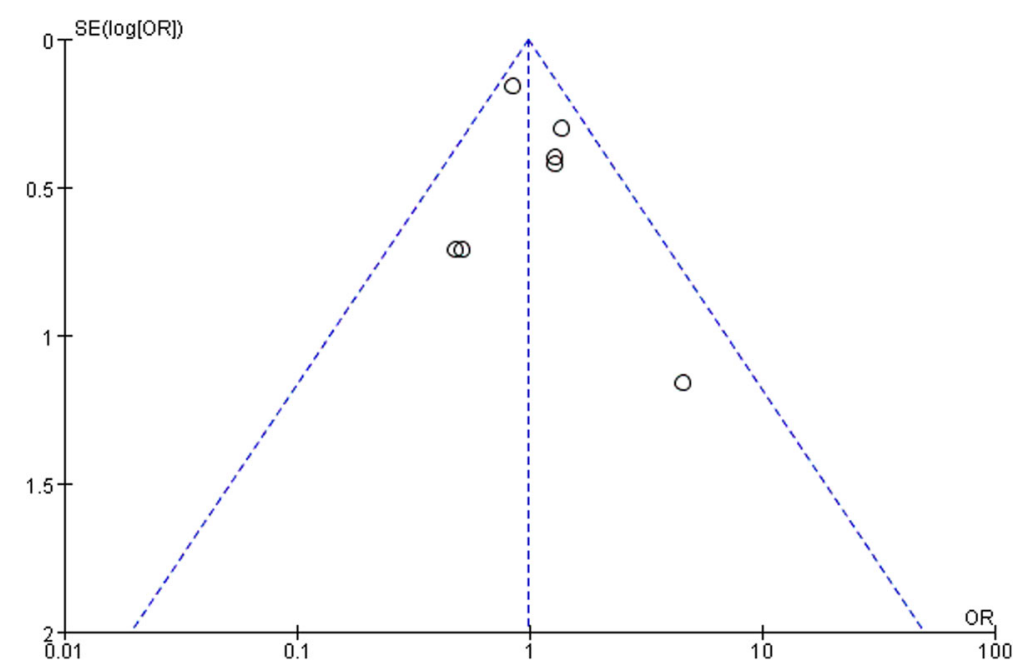

Fig. 4 Funnel plot of the mortality demonstrating that no publication bias existed 


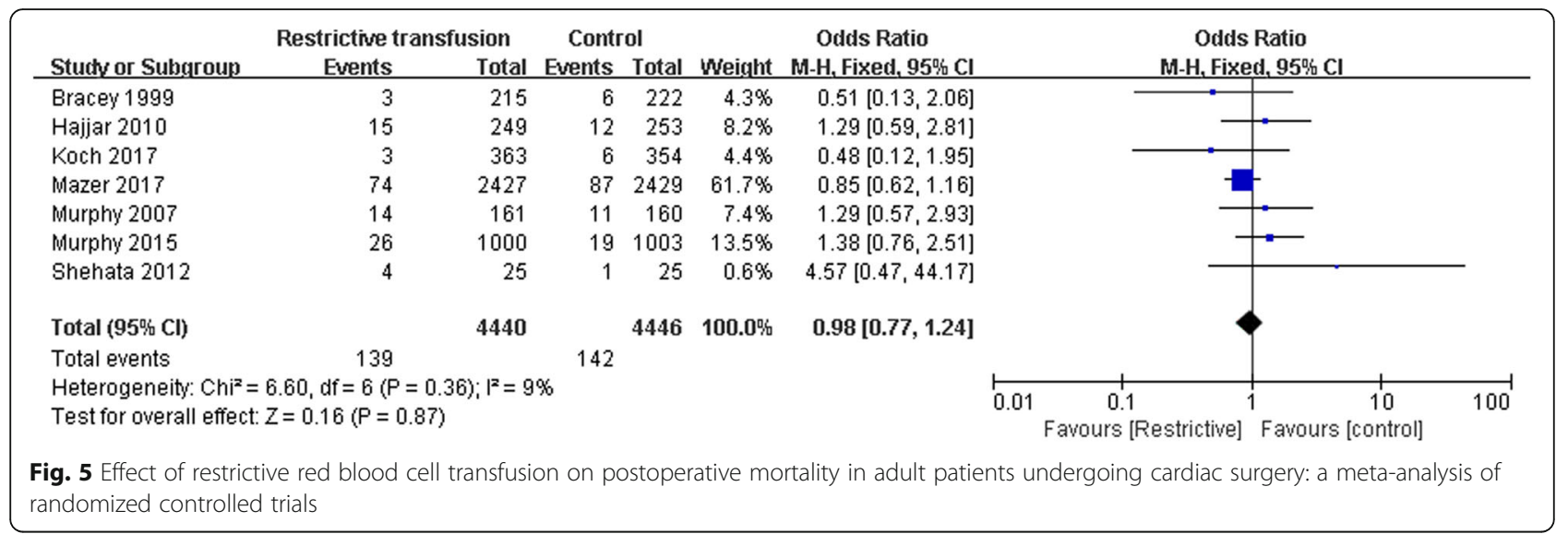

PBM programs included preoperative optimization of hemoglobin levels, blood-sparing techniques, and standardization of transfusion practice [28, 29]. Since then the PBM program has been adopted to minimize blood loss in patients undergoing cardiac surgeries [30]. Gross et al. [31] reported that implementing meticulous surgical techniques, a goal-directed coagulation algorithm, and a more restrictive transfusion threshold in combination resulted in an obvious decrease in RBC transfusions and lower total direct costs. Despite the benefits of PBM, many barriers limit translation of PBM guidelines into clinical practice worldwide, particularly in the absence of interdisciplinary commitment, lack of resources, and general concerns. Strategies for overcoming the obstacles include the use of bundles of care and specifically designed measures on the basis of local conditions [32].

Several pharmacologic agents have been used to decrease intraoperative blood loss, which is helpful to reduce RBC transfusion. Antifbrinolytic agents, including tranexamic acid and epsilon aminocaproic acid, have been extensively studied, and they decrease hemostatic activation, reduce bleeding, and decrease allogeneic $\mathrm{RBC}$ transfusions [33, 34]. Furthermore hemostatic treatment with fibrinogen concentrate in patients undergoing aortic surgery significantly reduced allogeneic blood transfusion [35]. In addition, several erythropoietin dosing regimens and duration treatment increase red cell mass and reduce allogeneic blood transfusions [36]. Erythropoietin administered before cardiac surgery seems effective in reducing the need for RBCs without increasing adverse events, hence reducing transfusion requirements [37, 38]; however, it is still controversial [39]. Recently Urena et al. [40] showed that combined erythropoietin and iron therapy failed to reduce $\mathrm{RBC}$ transfusion in anemic patients undergoing cardiac surgery.

This meta-analysis has several limitations. First, the hemoglobin thresholds of the restrictive RBC transfusion strategies varied between the trials. Thus, the appropriate threshold remains to be defined and could vary for different patients. Second, the types of cardiac surgery differed among the included studies and patients undergoing different types of cardiac surgery may have different tolerances to restrictive transfusion strategies.

\section{Conclusions}

The available evidence from our updated meta-analysis suggests that the OR for 30-day mortality did not favor a restrictive or liberal transfusion strategy in randomized controlled trials of adult patients undergoing cardiac surgery. Our meta-analysis is the best available evidence that restrictive RBC transfusion is as effective and safe as liberal transfusion strategies in adult cardiac surgery,

Table 2 Effects of red blood cell transfusion by outcome

\begin{tabular}{|c|c|c|c|c|c|c|}
\hline & Number of studies & Number of patients & $\begin{array}{l}\text { Fixed effects Odds ratio } \\
(95 \% \mathrm{Cl})\end{array}$ & Fixed effects $p$ value & $I^{2}(\%)$ & Heterogeneity $p$ value \\
\hline Mortality & 7 & 8886 & $0.98(0.77-1.24)$ & 0.87 & 9 & 0.36 \\
\hline Pulmonary morbidity & 5 & 3658 & $1.09(0.88-1.34)$ & 0.42 & 0 & 0.44 \\
\hline AKI & 6 & 8355 & $1.03(0.92-1.14)$ & 0.65 & 0 & 0.71 \\
\hline AMI & 4 & 7302 & $1.01(0.80-1.27)$ & 0.95 & 0 & 0.78 \\
\hline Infectious morbidity & 6 & 8444 & $1.11(0.95-1.3)$ & 0.19 & 0 & 0.58 \\
\hline Cerebrovascular accident & 6 & 8528 & $0.97(0.72-1.30)$ & 0.84 & 0 & 0.66 \\
\hline
\end{tabular}

$A K I$ acute kidney injury, $A M I$ acute myocardial infarction 
although the appropriate threshold remains to be defined and could vary for different patients.

\section{Additional files}

Additional file 1: Effect of restrictive red blood cell transfusion on pulmonary morbidity. Forest plot of adult patients undergoing cardiac surgery. Pulmonary morbidity includes acute respiratory distress syndrome, acute lung injury, delayed extubation. ARDS and ALI are according to the Berlin definition. Delayed extubation defined by inability to extubate the patients within $24 \mathrm{~h}$ after the completion of the surgical procedure. (PNG $5 \mathrm{~kb}$ )

Additional file 2: Effect of restrictive red blood cell transfusion on postoperative acute kidney injury (AKI). Forest plot of adult patients undergoing cardiac surgery. AKI is defined according to the KDIGO or RIFLE criteria or as dialysis-dependent or $50 \%$ or greater increase in serum creatinine. (PNG $6 \mathrm{~kb}$ )

Additional file 3: Effect of restrictive red blood cell transfusion on postoperative infections. Forest plot in adult patients undergoing cardiac surgery. Pneumonia was defined as autopsy diagnosis or roentgenographic infiltrate and at least two of the following three criteria: fever, leukocytosis, and positive sputum culture; or deep sternal or leg wound infection requiring intravenous antibiotics and/or surgical debridement. (PNG $6 \mathrm{~kb}$ )

Additional file 4: Effect of restrictive red blood cell transfusion on postoperative acute myocardial infarction (AMI). Forest plot of adult patients undergoing cardiac surgery. Myocardial infarction was defined according to the task force for the European Society of Cardiology, the American College of Cardiology Foundation, the American Heart Association, and the World Heart Federation. (PNG 5 kb)

Additional file 5: Effect of restrictive red blood cell transfusion on postoperative cerebrovascular accident. Forest plot of adult patients undergoing cardiac surgery. Cerebrovascular accident is defined as new focal neurological deficit lasting more than $24 \mathrm{~h}$ confirmed by clinical assessment and brain imaging. (PNG $6 \mathrm{~kb}$ )

\section{Abbreviations}

AKI: Acute kidney injury; AMI: Acute myocardial infarction; CABG: Coronary artery bypass grafting; CARE: Cardiac anesthesia risk score; EuroSCORE: European System for Cardiac Operative Risk Evaluation; Hb: Hemoglobin; HVR: Heart valve replacement; ICU: Intensive care unit: RBC: Red blood cell; TSA: Trial sequential analysis

\section{Funding}

Contract grant sponsor: National Natural Science Foundations of China; contract grant number 81670065 .

Social Development Funds of Jiangsu Province; contract grant number BE2017691. Social Development Funds of Yangzhou City; contract grant number YZ2017086. Jiangsu Provincial Medical Youth Talent; xcontract grant number QNRC2016317.

\section{Availability of data and materials}

The datasets used and/or analyzed in the current study are available from the corresponding author upon reasonable request.

\section{Authors' contributions}

QC designed the study, performed the data analysis, and drafted the manuscript. HW participated in the quality assessment and the design of the study and helped to revise the manuscript. LL participated in the conception and design of the study, performed the literature search, and helped to revise the manuscript for important intellectual content. JS performed the literature search, quality assessment, and data analysis, and helped to revise the manuscript. JY participated in the quality assessment of the study, helped to draft the manuscript, and performed the statistical analysis. RZ performed the data analysis and statistical analysis and helped to revise the manuscript. All authors have read and approved the final manuscript.

\section{Ethics approval and consent to participate} Not applicable.
Consent for publication

All authors have agreed to the publication of this manuscript.

Competing interests

The authors declare that they have no competing interests.

\section{Publisher's Note}

Springer Nature remains neutral with regard to jurisdictional claims in published maps and institutional affiliations.

\section{Author details}

'Department of Critical Care Medicine, Northern Jiangsu People's Hospital; Clinical Medical College, Yangzhou University, 98 Nantong West Road, Yangzhou 225001, People's Republic of China. ' ${ }^{2}$ Department of Cardiology, Northern Jiangsu People's Hospital; Clinical Medical College, Yangzhou University, 98 Nantong West Road, Yangzhou 225001, People's Republic of China.

Received: 1 January 2018 Accepted: 10 May 2018

Published online: 31 May 2018

\section{References}

1. Duque-Sosa P, Martínez-Urbistondo D, Echarri G, et al. Perioperative hemoglobin area under the curve is an independent predictor of renal failure after cardiac surgery. Results from a Spanish multicenter retrospective cohort study. PLoS One. 2017;12(2):e0172021.

2. von Heymann C, Kaufner L, Sander M, et al. Does the severity of preoperative anemia or blood transfusion have a stronger impact on longterm survival after cardiac surgery? J Thorac Cardiovasc Surg. 2016;152(5): 1412-20.

3. Karkouti K, Wijeysundera DN, Beattie WS. Risk associated with preoperative anemia in cardiac surgery: a multicenter cohort study. Circulation. 2008; 117(4):478-84.

4. Bennett-Guerrero E, Zhao Y, O'Brien SM, et al. Variation in use of blood transfusion in coronary artery bypass graft surgery. JAMA. 2010;304(14): 1568-75.

5. Wells AW, Llewelyn CA, Casbard A, et al. The EASTR Study: indications for transfusion and estimates of transfusion recipient numbers in hospitals supplied by the National Blood Service. Transfus Med. 2009;19(6):315-28.

6. Horvath KA, Acker MA, Chang $\mathrm{H}$, et al. Blood transfusion and infection after cardiac surgery. Ann Thorac Surg. 2013;95(6):2194-201.

7. Murphy GJ, Reeves BC, Rogers CA, et al. Increased mortality, postoperative morbidity, and cost after red blood cell transfusion in patients having cardiac surgery. Circulation. 2007:116(22):2544-52.

8. Shaw RE, Johnson CK, Ferrari G, et al. Blood transfusion in cardiac surgery does increase the risk of 5-year mortality: results from a contemporary series of 1714 propensity-matched patients. Transfusion. 2014;54(4):1106-13.

9. Mazer CD, Whitlock RP, Fergusson DA, et al. Restrictive or liberal red-cell transfusion for cardiac surgery. N Engl J Med. 2017;377(22):2133-44.

10. Koch CG, Sessler DI, Mascha EJ, et al. A randomized clinical trial of red blood cell transfusion triggers in cardiac surgery. Ann Thorac Surg. 2017; 104(4):1243-50.

11. Chen $\mathrm{QH}$, Zheng $\mathrm{RQ}$, Lin $\mathrm{H}$, et al. Effect of levosimendan on prognosis in adult patients undergoing cardiac surgery: a meta-analysis of randomized controlled trials. Crit Care. 2017;21(1):253.

12. Bracey AW, Radovancevic R, Riggs SA, et al. Lowering the hemoglobin threshold for transfusion in coronary artery bypass procedures: effect on patient outcome. Transfusion. 1999;39(10):1070-7.

13. Hajiar LA, Vincent JL, Galas FR, et al. Transfusion requirements after cardiac surgery: the TRACS randomized controlled trial. JAMA. 2010;304(14):1559-67.

14. Murphy GJ, Pike K, Rogers CA, et al. Liberal or restrictive transfusion after cardiac surgery. N Engl J Med. 2015;372(11):997-1008.

15. Shehata N, Burns LA, Nathan $H$, et al. A randomized controlled pilot study of adherence to transfusion strategies in cardiac surgery. Transfusion. 2012; 52(1):91-9.

16. Murphy GJ, Rizvi SI, Battaglia F, et al. A pilot randomized controlled trial of the effect of transfusion-threshold reduction on transfusion rates and morbidity after cardiac surgery. Transfus Altern Transfus Med. 2007;9(suppl 1):41-2. 
17. Koch CG, Li L, Duncan Al, et al. Transfusion in coronary artery bypass grafting is associated with reduced long-term survival. Ann Thorac Surg. 2006;81(5):1650-7.

18. Huynh K. Surgery: restrictive versus liberal red-cell transfusion. Nat Rev Cardiol. 2018;15(1):2.

19. Laine A, Niemi T, Schramko A. Transfusion threshold of hemoglobin $80 \mathrm{~g} / \mathrm{L}$ is comparable to $100 \mathrm{~g} / \mathrm{L}$ in terms of bleeding in cardiac surgery: a prospective randomized study. J Cardiothorac Vasc Anesth. 2018;32(1):131-139.

20. Patel NN, Avlonitis VS, Jones HE, et al. Indications for red blood cell transfusion in cardiac surgery: a systematic review and meta-analysis. Lancet Haematol. 2015;2(12):e543-53.

21. Simon Gl, Craswell A, Thom O, et al. Outcomes of restrictive versus liberal transfusion strategies in older adults from nine randomised controlled trials: a systematic review and meta-analysis. Lancet Haematol. 2017;4(10):e465-74.

22. Nakamura RE, Vincent JL, Fukushima JT, et al. A liberal strategy of red blood cell transfusion reduces cardiogenic shock in elderly patients undergoing cardiac surgery. J Thorac Cardiovasc Surg. 2015;150(5):1314-20.

23. Cortés-Puch I, Wiley BM, Sun J, et al. Risks of restrictive red blood cell transfusion strategies in patients with cardiovascular disease (CVD): a meta-analysis. Transfus Med. 2018; https:/doi.org/10.1111/tme.12535. [Epub ahead of print]

24. Kuduvalli $\mathrm{M}$, Oo AY, Newall $\mathrm{N}$, et al. Effect of peri-operative red blood cell transfusion on 30-day and 1-year mortality following coronary artery bypass surgery. Eur J Cardiothorac Surg. 2005;27(4):592-8.

25. Andreasen JJ, Dethlefsen C, Modrau IS, et al. Storage time of allogeneic red blood cells is associated with risk of severe postoperative infection after coronary artery bypass grafting. Eur J Cardiothorac Surg. 2011;39(3):329-34

26. Putney L. Bloodless cardiac surgery: not just possible, but preferable. Crit Care Nurs Q. 2007:30(3):263-70.

27. Farmer SL, Towler SC, Leahy MF, et al. Drivers for change: Western Australia Patient Blood Management Program (WA PBMP), World Health Assembly (WHA) and Advisory Committee on Blood Safety and Availability (ACBSA). Best Pract Res Clin Anaesthesiol. 2013;27(1):43-58.

28. Spahn DR, Goodnough LT. Alternatives to blood transfusion. Lancet. 2013; 381(9880):1855-65.

29. Mehra T, Seifert B, Bravo-Reiter S, et al. Implementation of a patient blood management monitoring and feedback program significantly reduces transfusions and costs. Transfusion. 2015;55(12):2807-15.

30. Meybohm P, Herrmann E, Steinbicker AU, et al. Patient blood management is associated with a substantial reduction of red blood cell utilization and safe for patient's outcome: a prospective, multicenter cohort study with a noninferiority design. Ann Surg. 2016;264(2):203-11.

31. Gross I, Seifert B, Hofmann A, et al. Patient blood management in cardiac surgery results in fewer transfusions and better outcome. Transfusion. 2015; 55(5):1075-81.

32. Meybohm P, Richards T, Isbister J, et al. Patient blood management bundles to facilitate implementation. Transfus Med Rev. 2017 Jan;31(1):62-71.

33. Henry D, Carless P, Fergusson D, et al. The safety of aprotinin and lysinederived antifibrinolytic drugs in cardiac surgery: a meta-analysis. CMAJ. 2009; 180(2):183-93.

34. Koster A, Faraoni D, Levy JH. Antifibrinolytic therapy for cardiac surgery: an update. Anesthesiology. 2015;123(1):214-21.

35. Rahe-Meyer N, Solomon C, Hanke A, et al. Effects of fibrinogen concentrate as first-line therapy during major aortic replacement surgery: a randomized, placebo-controlled trial. Anesthesiology. 2013;118(1):40-50.

36. Corwin $\mathrm{HL}$, Gettinger A, Rodriguez RM, et al. Efficacy of recombinant human erythropoietin in the critically ill patient: a randomized, double-blind, placebo-controlled trial. Crit Care Med. 1999 Nov;27(11):2346-50.

37. Yoo YC, Shim JK, Kim JC, et al. Effect of single recombinant human erythropoietin injection on transfusion requirements in preoperatively anemic patients undergoing valvular heart surgery. Anesthesiology. 2011; 115(5):929-37.

38. Alghamdi AA, Albanna MJ, Guru V, et al. Does the use of erythropoietin reduce the risk of exposure to allogeneic blood transfusion in cardiac surgery? A systematic review and meta-analysis. J Card Surg. 2006;21(3):320-6.

39. Weltert $L$, Rondinelli B, Bello R, et al. A single dose of erythropoietin reduces perioperative transfusions in cardiac surgery: results of a prospective singleblind randomized controlled trial. Transfusion. 2015;55(7):1644-54.

40. Urena M, Del Trigo M, Altisent OA, et al. Combined erythropoietin and iron therapy for anaemic patients undergoing transcatheter aortic valve implantation: the EPICURE randomised clinical trial. Euro Intervention. 2017; 13(1):44-52. 